\title{
Semilocal exchange hole with an application to range-separated density functionals
}

\author{
Jianmin Tao, ${ }^{1, *}$ Ireneusz W. Bulik, ${ }^{2}$ and Gustavo E. Scuseria ${ }^{2}$ \\ ${ }^{1}$ Department of Physics, Temple University, Philadelphia, Pennsylvania 19122-1801, USA \\ ${ }^{2}$ Department of Chemistry and Department of Physics and Astronomy, Rice University, Houston, Texas 77005, USA
}

(Received 14 November 2016; revised manuscript received 20 February 2017; published 13 March 2017)

\begin{abstract}
The exchange-correlation hole is a central concept in density functional theory. It not only provides justification for an exchange-correlation energy functional but also serves as a local ingredient for nonlocal range-separated density functionals. However, due to the nonlocal nature, modeling the conventional exact exchange hole presents a great challenge to density functional theory. In this work, we propose a semilocal exchange hole underlying the Tao-Perdew-Staroverov-Scuseria (TPSS) meta-generalized gradient approximation functional. Our model is distinct from previous ones not only at small separation between an electron and the hole around the electron but also in the way it interpolates between rapidly varying and slowly varying densities. Here the interpolation is determined by the wave-vector analysis on the infinite-barrier model for a jellium surface. Numerical tests show that our exchange-hole model mimics the conventional exact one quite well for atoms. As a simple application, we apply the hole model to construct a TPSS-based range-separated functional. We find that this range-separated functional can substantially improve the band gaps and barrier heights of TPSS, without losing much accuracy for atomization energies.
\end{abstract}

DOI: 10.1103/PhysRevB.95.125115

\section{INTRODUCTION}

Kohn-Sham density functional theory (DFT) $[1-3]$ is a mainstream electronic structure theory due to its useful accuracy and high computational efficiency. Formally, it is an exact theory, but in practice the exchange-correlation energy component, which accounts for all many-body effects, has to be approximated as a functional of the electron density. Development of exchange-correlation energy functionals for a wide class of problems with high accuracy has been the central task of DFT. Many density functionals have been proposed [4-24], and some of them have achieved remarkable accuracy in condensed-matter physics or quantum chemistry or both.

According to their local ingredients, density functionals can be classified into two broad categories: semilocal and nonlocal. Semilocal functionals make use of the local electron density, density derivatives, and/or the orbital kinetic energy density as inputs, such as the local spin-density approximation (LSDA) $[25,26]$, generalized gradient approximation (GGA) [10,27,28], and meta-GGA [11,16,17,20,24]. Due to the simplicity in theoretical construction and numerical implementation, as well as relatively low computational cost, semilocal functionals have been widely used in electronic structure calculations [29-32]. Indeed, semilocal DFT can give a quick and often accurate prediction of many properties such as enthalpies of formation or atomization energies [23,33-38], bond lengths [39,40], lattice constants [40-44], cohesive energies [45], etc.

Semilocal DFT has achieved a high level of sophistication and practical success for many problems in chemistry, physics, and materials science, but it encounters difficulty in the prediction of reaction barrier heights, band gaps, charge transfer, and excitation energies. Accurate description of these properties requires electronic nonlocality [46], which is absent

\footnotetext{
*Corresponding author: jianmin.tao@ temple.edu; http://www.sas. upenn.edu/ jianmint/
}

in semilocal functionals. Nonlocality can be accounted for via mixing some amount of exact exchange into a semilocal DFT. This leads to the development of hybrid $[8,13,33,47]$ and range-separated functionals [14,48]. The former involve the exact exchange energy or energy density, while the latter involve the exact and approximate semilocal exchange holes.

There are three ways to approximate an exchange hole. It can be constructed from paradigm densities in which the exact exchange hole is known, such as the slowly varying density $[4,49,50]$ (the paradigm of condensed-matter physics) and the one-electron density [7] (the paradigm of quantum chemistry). It can also be constructed from a density functional with the reverse-engineering approach [51-53]. A physically more appealing approach to approximate an exchange hole is from the density-matrix expansion [24]. Among the three general methods, the reverse-engineering approach is most frequently used. However, a semilocal exchange hole based on the reverse-engineering approach may not be in the gauge of the conventional exchange hole because a semilocal exchange energy density is usually not in the conventional gauge [54]. In the construction of a semilocal exchange hole, one must impose certain exact constraints on a hole to recover the underlying exchange energy density, which is usually not in the same gauge of the conventional exchange energy density, due to the integration by parts performed in the construction of semilocal DFT. Examples include the Perdew-Burke-Ernzerhof (PBE) GGA [49,51] and Tao-Perdew-Staroverov-Scuseria (TPSS) meta-GGA [52,53] exchange holes. Many range-separated functionals have been proposed [14,55-58], and some of them have obtained great popularity in electronic structure calculations.

The exchange hole in the conventional gauge is of special interest. For example, the subsystem functional scheme proposed by Mattsson and coworkers [15,59-61] was developed from the conventional exchange hole of the edge electron gas [62]. In the present work, we aim to develop an exchange hole in the conventional gauge. The hole will reproduce the TPSS exchange energy functional by construction. To 
ensure that our model hole is in the conventional gauge, we not only impose the exact conventional constraints in the conventional gauge (e.g., recovery of the correct short-range behavior without integration by parts) on the hole model but also modify the TPSS exchange energy density by adding a gauge function. The present gauge function is similar to the one proposed by Tao et al. [54], but with a modification so that the gauge-corrected exchange energy density or underlying hole is ensured to be negative even in the far density tail. Adding a proper gauge function to the exchange energy density will not alter the integrated exchange energy, but it will improve the agreement of the model hole with the exact conventional one. Furthermore, the hole model can generate the exact system-averaged exchange hole accurately by replacing the TPSS exchange energy density with the gauge-corrected exact conventional exchange energy density (i.e., in TPSS gauge). As a simple application, we apply our semilocal exchange hole to construct a range-separated exchange functional. Our numerical tests show that this range-separated functional, when combined with the TPSS correlation functional, can yield band gaps and barrier heights in much better agreement with experimental values than the original TPSS functional, without losing much accuracy of atomization energies.

\section{EXACT CONVENTIONAL EXCHANGE HOLE}

For simplicity, let us first consider a spin-unpolarized density $\left(n_{\uparrow}=n_{\downarrow}\right)$. For such a density, the exchange energy can be written as

$$
\begin{aligned}
E_{\mathrm{x}}[n] & =\int d^{3} r n(\mathbf{r}) \epsilon_{\mathrm{x}}(\mathbf{r}) \\
& =\int d^{3} r n(\mathbf{r}) \frac{1}{2} \int d^{3} u \frac{\rho_{\mathrm{x}}(\mathbf{r}, \mathbf{r}+\mathbf{u})}{u},
\end{aligned}
$$

where $n(\mathbf{r})=n_{\uparrow}+n_{\downarrow}$ is the total electron density, $\epsilon_{\mathrm{x}}(\mathbf{r})$ is the conventional exchange energy per electron, or, loosely speaking, the exchange energy density, and $\rho_{\mathrm{x}}(\mathbf{r}, \mathbf{r}+\mathbf{u})$ is the exchange hole at $\mathbf{r}+\mathbf{u}$ around an electron at $\mathbf{r}$. It is conventionally defined by

$$
\rho_{\mathrm{x}}(\mathbf{r}, \mathbf{r}+\mathbf{u})=-\left|\gamma_{1}(\mathbf{r}, \mathbf{r}+\mathbf{u})\right|^{2} / 2 n(\mathbf{r}) .
$$

Here $\gamma_{1}(\mathbf{r}, \mathbf{r}+\mathbf{u})$ is the Kohn-Sham single-particle density matrix given by

$$
\gamma_{1}(\mathbf{r}, \mathbf{r}+\mathbf{u})=2 \sum_{i}^{N / 2} \phi_{i}(\mathbf{r})^{*} \phi_{i}(\mathbf{r}+\mathbf{u}),
$$

with $N$ being the number of electrons and $\phi_{i}(\mathbf{r})$ being the occupied Kohn-Sham orbitals. According to expression (1), one can regard the exchange energy as the electrostatic interaction between a reference electron at $\mathbf{r}$ and the exchange hole at $\mathbf{r}+\mathbf{u}$. Therefore, strictly speaking, an exchange energy functional cannot be fully justified unless the underlying exchange hole has been found. But this issue can be addressed with the reverse-engineering approach [52].

The exchange hole for a spin-unpolarized density can be generalized to any spin polarization with the spin-scaling relation [63]

$$
\rho_{\mathrm{x}}\left[n_{\uparrow}, n_{\downarrow}\right]=\frac{n_{\uparrow}}{n} \rho_{\mathrm{x}}\left[2 n_{\uparrow}\right]+\frac{n_{\downarrow}}{n} \rho_{\mathrm{x}}\left[2 n_{\downarrow}\right] .
$$

Therefore, in the development of the exchange hole, we need to consider only a spin-compensated density. Performing the spherical average of the exchange hole over the direction of separation vector $\mathbf{u}$, the exchange energy of Eq. (1) may be rewritten as

$$
E_{\mathrm{x}}[n]=\int_{0}^{\infty} d u 4 \pi u^{2} \int d^{3} r n(\mathbf{r}) \frac{\left\langle\rho_{\mathrm{x}}(\mathbf{r}, u)\right\rangle_{\mathrm{sph}}}{2 u},
$$

where $\left\langle\rho_{\mathrm{x}}(\mathbf{r}, u)\right\rangle_{\mathrm{sph}}$ is the spherical average of the exchange hole defined by

$$
\left\langle\rho_{\mathrm{x}}(\mathbf{r}, u)\right\rangle_{\mathrm{sph}}=\int \frac{d \Omega_{\mathbf{u}}}{4 \pi} \rho_{\mathrm{x}}(\mathbf{r}, \mathbf{r}+\mathbf{u}) .
$$

This suggests that the exchange energy does not depend on the detail of the associated hole. Rearranging Eq. (5) leads to a simple expression

$$
E_{\mathrm{x}}[n]=N \int d u 4 \pi u^{2} \frac{\left\langle\rho_{\mathrm{x}}(u)\right\rangle}{2 u},
$$

where $\left\langle\rho_{\mathrm{xc}}(u)\right\rangle$ is the system average of the exchange hole defined by

$$
\left\langle\rho_{\mathrm{x}}(u)\right\rangle=\frac{1}{N} \int d^{3} r n(\mathbf{r})\left\langle\rho_{\mathrm{x}}(\mathbf{r}, u)\right\rangle_{\mathrm{sph}} .
$$

Although the conventional exact exchange hole of Eq. (2) satisfies the sum rule

$$
\int d^{3} u \rho_{\mathrm{x}}(\mathbf{r}, \mathbf{u})=-1
$$

(the most important property of the exchange hole), the exact exchange hole transformed to a new coordinate system $[64,65]$ does not. Nevertheless, the system-averaged hole always satisfies the sum rule

$$
\int d^{3} u\left\langle\rho_{\mathrm{x}}(u)\right\rangle=-1 .
$$

This is the constraint that has been imposed in the development of a semilocal exchange hole. While the exchange energy is uniquely defined, the exchange energy density $\epsilon_{\mathrm{x}}(\mathbf{r})$ as well as the exchange hole $\rho_{\mathrm{x}}(\mathbf{r}, \mathbf{r}+\mathbf{u})$ are not. For example, both quantities can be altered by a general coordinate transformation or by adding an arbitrary amount of the Laplacian of the electron density, without changing the total exchange energy $[54,66]$.

\section{CONSTRAINTS ON THE EXCHANGE HOLE}

The conventional exchange hole is related to the pair distribution function $g_{x}\left(\mathbf{r}, \mathbf{r}^{\prime}\right)$ by

$$
n(\mathbf{r}) \rho_{\mathrm{x}}\left(\mathbf{r}, \mathbf{r}^{\prime}\right)=n(\mathbf{r}) n\left(\mathbf{r}^{\prime}\right) g_{x}\left(\mathbf{r}, \mathbf{r}^{\prime}\right) .
$$

In general, a semilocal exchange hole can be written as

$$
n(\mathbf{r}) \rho_{\mathrm{x}}(\mathbf{r}, \mathbf{r}+u)=n^{2}(\mathbf{r}) J_{\mathrm{x}}\left(s, z, u_{f}\right),
$$

where $J\left(s, z, u_{f}\right)$ is the shape function that needs to be constructed, with $s=|\nabla n| /\left(2 k_{f} n\right)$ being the dimensionless reduced density gradient, $k_{f}=\left(3 \pi^{2} n\right)^{1 / 3}$ being the Fermi wave vector, $z=\tau_{W} / \tau$, and $u_{f}=k_{f} u$. Here $\tau_{W}=|\nabla n|^{2} / 8 n$ 
is the von Weizsäcker kinetic energy density, and $\tau$ is the Kohn-Sham orbital kinetic energy density defined by $\tau(\mathbf{r})=$ $\sum_{i}^{N / 2}\left|\nabla \phi_{i}(\mathbf{r})\right|^{2}$.

\section{A. Constraints on the shape function}

We will seek a shape function that satisfies the following constraints:

(i) On-top value

$$
J(s, z, 0)=-1 / 2 .
$$

(ii) Uniform-gas limit

$$
J^{\mathrm{unif}}\left(u_{f}\right)=-\frac{9}{2}\left[\frac{\sin \left(u_{f}\right)-\cos \left(u_{f}\right)}{u_{f}^{3}}\right] .
$$

The uniform-gas limit that will be imposed here is the nonoscillatory model [67] [Eq. (28) for $s=0$ and $z=0$ ].

(iii) Normalization

$$
\frac{4}{3 \pi} \int_{0}^{\infty} d u_{f} u_{f}^{2} J\left(s, z, u_{f}\right)=-1 .
$$

(iv) Negativity

$$
J\left(s, z, u_{f}\right) \leqslant 0 .
$$

(v) Energy constraint

$$
\frac{8}{9} \int_{0}^{\infty} d u_{f} u_{f} J\left(s, z, u_{f}\right)=-F_{x}^{\mathrm{TPSS}}(s, z) .
$$

(vi) Small- $u$ behavior

$$
\lim _{u_{f} \rightarrow 0} \frac{\partial^{2} J\left(s, z, u_{f}\right)}{\partial u_{f}^{2}}=L(s, z) .
$$

$L(s, z)$ is the curvature of the shape function that will be discussed below.

(vii) Large-gradient limit

$$
\lim _{s \rightarrow \infty} J\left(s, z, u_{f}\right)=J^{\mathrm{PBE}}\left(s, u_{f}\right) .
$$

In the large-gradient limit, the TPSS enhancement factor approaches the PBE enhancement factor. Therefore, the TPSS shape function should also approach the PBE shape function in this limit.

Among these constraints, (vi) is for the conventional exchange hole, while (vii) is a constraint used in the development of the TPSS functional. These two constraints will be discussed in detail below. In previous works [52,53], constraint (vi) was used with integration by parts and thus is not a constraint for the conventional exchange hole, and constraint (vii) was not considered.

\section{B. Small- $u$ behavior and large-gradient limit}

Expanding the spherically averaged exchange hole up to second order in $u$ yields

$\left\langle\rho_{x}(\mathbf{r}, u)\right\rangle_{\mathrm{sph}}=-\frac{1}{2} n+\frac{1}{12}\left[4\left(\tau-\frac{|\nabla n|^{2}}{8 n}\right)-\nabla^{2} n\right] u^{2}+\cdots$.

Since the Laplacian of the density tends to negative infinity at a nucleus, the negativity of the exchange hole for small $u$ will be violated. Therefore, we must eliminate it. In previous works, the Laplacian of the density is eliminated by integration by parts [52]. In order to model the conventional exchange hole, here we eliminate it instead with the second-order gradient expansion of the kinetic energy density in the slowly varying limit,

$$
\tau \approx \tau^{\mathrm{unif}}+|\nabla n|^{2} /(72 n)+\nabla^{2} n / 6
$$

This technique has been used in the development of the TPSS [17] and other functionals [18,68] as well as in the construction of electron localization indicator [69].

Substituting Eqs. (20) into Eq. (12) and eliminating the Laplacian $\nabla^{2} n$ via (21) yields the small- $u$ expansion of the shape function

$$
J\left(s, z, u_{f}\right)=-\frac{1}{2}+\frac{1}{6}\left(-\frac{3}{10} \frac{\tau}{\tau^{\mathrm{uni}}}+\frac{9}{10}-\frac{5}{6} s^{2}\right) u_{f}^{2}+\cdots,
$$

leading to

$$
L(s, z)=-\frac{1}{3}\left(\frac{3}{10} \frac{\tau}{\tau^{\text {uni }}}-\frac{9}{10}+\frac{5}{6} s^{2}\right) .
$$

For one- or two-electron densities, $L(s, z)$ reduces to

$$
L(s, z=1)=\frac{3}{2}\left(\frac{1}{5}-\frac{8}{27} s^{2}\right)
$$

while for the uniform gas, $L(s=0, z=0)=\frac{1}{5}$. Note that $\lim _{s \rightarrow 0} L(s, z=1)=3 / 10$, while $\lim _{s \rightarrow 0} L(s, z=0)=1 / 5$ (order-of-limit problem).

In the large-gradient limit, the TPSS shape function should recover the PBE shape function [Eq. (19)]. This requires that $L(s, z)$ must be merged smoothly with the PBE small- $u$ behavior,

$$
L^{\mathrm{PBE}}(s)=\left(\frac{1}{5}-\frac{2}{27} s^{2}\right)
$$

We can achieve this with

$$
\begin{aligned}
L^{\mathrm{TPSS}}= & \frac{1}{2} \operatorname{erfc}\left(\frac{s^{2}-s_{0}^{2}}{s_{0}}\right) L(s, z) \\
& +\left[1-\frac{1}{2} \operatorname{erfc}\left(\frac{s^{2}-s_{0}^{2}}{s_{0}}\right)\right] L^{\mathrm{PBE}}(s),
\end{aligned}
$$

where $\operatorname{erfc}(x)$ is the complementary error function defined by

$$
\operatorname{erfc}(x)=1-\operatorname{erf}(x)=\frac{2}{\pi} \int_{x}^{\infty} d t e^{-t^{2}}
$$

Here $s_{0}=6$ is a switching parameter that defines the point at which the small- $u$ behavior smoothly changes from the TPSS to PBE. This choice of $s_{0}$ ensures that the small- $u$ behavior of our shape function is essentially determined by Eq. (22), while it merges into the PBE shape function in the large-gradient limit [Eqs. (35) and (36) of Ref. [67]]. 


\section{SHAPE FUNCTION FOR THE TPSS EXCHANGE HOLE}

\section{A. TPSS shape function}

The shape function for the TPSS exchange hole is assumed to take the following form:

$$
\begin{aligned}
J^{\mathrm{TPSS}}\left(u_{f} s, z\right)= & {\left[-\frac{9}{4 u_{f}^{4}}\left(1-e^{-A u_{f}^{2}}\right)\right.} \\
& +\left(\frac{9 A}{4 u_{f}^{2}}+B+C(s, z) u_{f}^{2}+G(s, z) u_{f}^{4}\right. \\
& \left.\left.+K(s, z) u_{f}^{6}\right) e^{-D u_{f}^{2}}\right] e^{-H(s, z) u_{f}^{2}}
\end{aligned}
$$

where $A=0.757211, B=-0.106364$, and $D=0.609650$ are determined by the recovery of the nonoscillatory model [67] of the uniform electron gas, while the functions $C(s, z), G(s, z)$, and $K(s, z)$ are determined by constraints (iii), (v), and (vi). They can be analytically expressed in terms of $H(s, z)$ as

$$
C=\frac{1}{8}\left(4 L+3 A^{3}+9 A^{2} H-9 A D^{2}-18 A D H+8 B \lambda\right),
$$

$$
\begin{aligned}
G= & -\frac{63}{8} \lambda^{3}\left[F_{x}^{\mathrm{TPSS}}+A \ln \left(\frac{\beta}{\lambda}\right)+H \ln \left(\frac{\beta}{H}\right)\right] \\
& -\frac{24}{5} \lambda^{\frac{7}{2}}\left(\frac{3 A}{\sqrt{H}+\sqrt{\beta}}-\sqrt{\pi}\right)+\frac{603}{40} A \lambda^{3} \\
& -\frac{19}{10} B \lambda^{2}-\frac{11}{10} C \lambda, \\
K= & \frac{8}{35} \lambda^{\frac{9}{2}}\left(\frac{3 A}{\sqrt{H}+\sqrt{\beta}}-\sqrt{\pi}\right)-\frac{12}{35} A \lambda^{4} \\
& -\frac{8}{105} B \lambda^{3}-\frac{4}{35} C \lambda^{2}-\frac{2}{7} G \lambda,
\end{aligned}
$$

where $\lambda=D+H(s, z)$ and $\beta=A+H(s, z)$. Following the procedure of Constantin, Perdew, and Tao [52] in the construction of the original TPSS shape function, here we determine the $s$ dependence of $H(s, z)$ by fitting to the two-electron exponential density, because for two-electron densities, $z$ is identically a constant everywhere in space. It depends only on the density gradient $s$. We determine the $z$ dependence of $H(s, z)$ with the wave-vector analysis of the surface energy in the infinite barrier model, because in this model, the electron density, the kinetic energy density, and the exchange hole are analytically known and the surface energy is also known accurately.

\section{B. $s$ dependence of $\mathrm{H}(s, z)$}

In iso-orbital regions where $z \approx 1$ (e.g., core and density tail regions), we assume that the function $H(s, z=1)$ takes the form

$$
H^{\text {iso-orb }}(s, z=1)=\frac{h_{0}+h_{1} s^{2}+h_{2} s^{4}+h_{3} s^{6}}{d_{0}+d_{1} s^{2}+d_{2} s^{4}+d_{3} s^{6}} .
$$

Note that $H^{\text {iso-orb }}(s, z)$ has only an even-order gradient dependence. This is because in the slowly varying limit, the spherical average of the exchange hole [Eq. (20)] depends only upon the even-order gradient terms [70]. In the large-gradient regime, $H(s, z=1)$ of TPSS should recover $H(s)$ [67] of PBE,

$$
H^{\mathrm{PBE}}(s)=\frac{p_{1} s^{2}+p_{2} s^{4}+p_{3} s^{6}}{1+p_{4} s^{2}+p_{5} s^{4}+p_{6} s^{6}} .
$$

For any density between the two regimes, we take the interpolation formula,

$$
\begin{aligned}
H(s, z=1)= & \frac{1}{2} \operatorname{erfc}\left(\frac{s^{2}-s_{0}^{2}}{s_{0}}\right) H^{\text {iso-orb }}(s, z=1) \\
& +\left[1-\frac{1}{2} \operatorname{erfc}\left(\frac{s^{2}-s_{0}^{2}}{s_{0}}\right)\right] H^{\mathrm{PBE}}(s) .
\end{aligned}
$$

Finally, we insert Eq. (34) into Eqs. (29)-(31) and perform the fitting procedure by minimizing the following quantity:

$$
\sum_{i} u_{i}\left(\left\langle\rho_{\mathrm{x}}^{\mathrm{TPSS}}\left(u_{i}\right)\right\rangle_{\mathrm{sph}}-\left\langle\rho_{\mathrm{x}}^{\mathrm{exact}}\left(u_{i}\right)\right\rangle_{\mathrm{sph}}\right)^{2},
$$

where $\left\langle\rho_{\mathrm{x}}(u)\right\rangle_{\mathrm{sph}}$ is the spherical system average of the exchange hole defined by Eq. (8). We can express $\left\langle\rho_{\mathrm{x}}(u)\right\rangle_{\mathrm{sph}}$ in terms of the shape function as $\left\langle\rho_{\mathrm{x}}(u)\right\rangle_{\mathrm{sph}}=$ $(1 / N) \int d^{3} r n(\mathbf{r})^{2} J\left(s, z, u_{f}\right)$. For numerical convenience, we replace the integral with discretized summation. All the parameters for $H(s, z=1)$ and $H(s)$ are listed in Table I.

Figure 1 shows the system-averaged exchange hole for the two-electron exponential density evaluated with different hole models compared to the exact one. We can observe from Fig. 1

\begin{tabular}{|c|c|c|c|c|c|c|c|c|c|c|c|c|c|}
\hline \multicolumn{8}{|c|}{$H(s, z=1)$ of Eq. (34) } & \multicolumn{6}{|c|}{$H(s)$ of Eq. (33) } \\
\hline$h_{0}$ & $h_{1}$ & $h_{2}$ & $h_{3}$ & $d_{0}$ & $d_{1}$ & $d_{2}$ & $d_{3}$ & $p_{1}$ & $p_{2}$ & $p_{3}$ & $p_{4}$ & $p_{5}$ & $p_{6}$ \\
\hline 0.0060 & 2.8916 & 0.7768 & 2.0876 & 13.695 & -0.2219 & 4.9917 & 0.7972 & 0.0302 & -0.1035 & 0.1272 & 0.1203 & 0.4859 & 0.1008 \\
\hline
\end{tabular}
that the present TPSS hole is slightly closer to the conventional exact hole than the original TPSS hole, but it is much closer than the PBE GGA and LSDA holes.

\section{Infinite barrier model and wave-vector analysis for surface energy}

As discussed above, in iso-orbital regions, the $s$ dependence of $H(s, z)$ is determined by fitting the model hole to the conventional exact exchange hole for the two-electron exponential density. In the uniform-gas limit, our exchange hole should correctly reduce to the nonoscillatory model [67] of the LSDA. This requires $H(s, z)$ to vanish in this limit. To

TABLE I. Parameters of the TPSS shape function $H(s, z=1)$ of Eq. (34) and the PBE shape function $H(s)$ of Eq. (33) determined by a fit to the two-electron exponential density. 


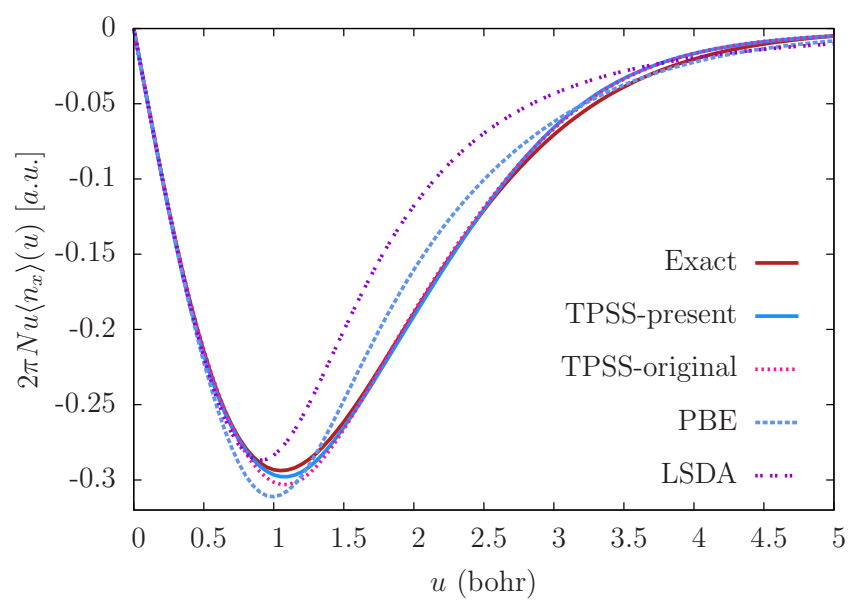

FIG. 1. System-averaged exchange hole for the LSDA, PBE GGA, and TPSS meta-GGA for the two-electron exponential density. "TPSS-original" represents the original TPSS hole model of Constantin, Perdew, and Tao [52], while "TPSS-present" represents the present TPSS hole model. The area under the curve is the exchange energy (in hartrees): $E_{\mathrm{x}}^{\mathrm{LSDA}}=-0.5361, E_{\mathrm{x}}^{\mathrm{PBE}}=-0.6117$, $E_{\mathrm{x}}^{\mathrm{TPSS}}=-0.6250$, and $E_{\mathrm{x}}^{\mathrm{ex}}=-0.6250$. Both the original and present TPSS holes yield the same exchange energy due to the same energy constraint.

fulfill these considerations, we assume that

$$
\begin{aligned}
H(s, z)= & \frac{1}{2} \operatorname{erfc}\left(\frac{s^{2}-s_{0}^{2}}{s_{0}}\right) H^{\text {iso-orb }}(s, z=1) z^{m} \\
& +\left[1-\frac{1}{2} \operatorname{erfc}\left(\frac{s^{2}-s_{0}^{2}}{s_{0}}\right)\right] H^{\mathrm{PBE}}(s),
\end{aligned}
$$

where $m$ is an integer. In order to determine $m$, we follow the procedure of Ref. [52] to study the wave-vector analysis (WVA) of the surface energy. But instead of using the jellium surface model with a linearly increasing barrier, here we employ the exactly solvable infinite barrier model (IBM). Since the single-particle density matrix and hence the electron density of IBM is analytically known, this allows us to obtain insight into the $z$ dependence of $H(s, z)$ from this model more easily.

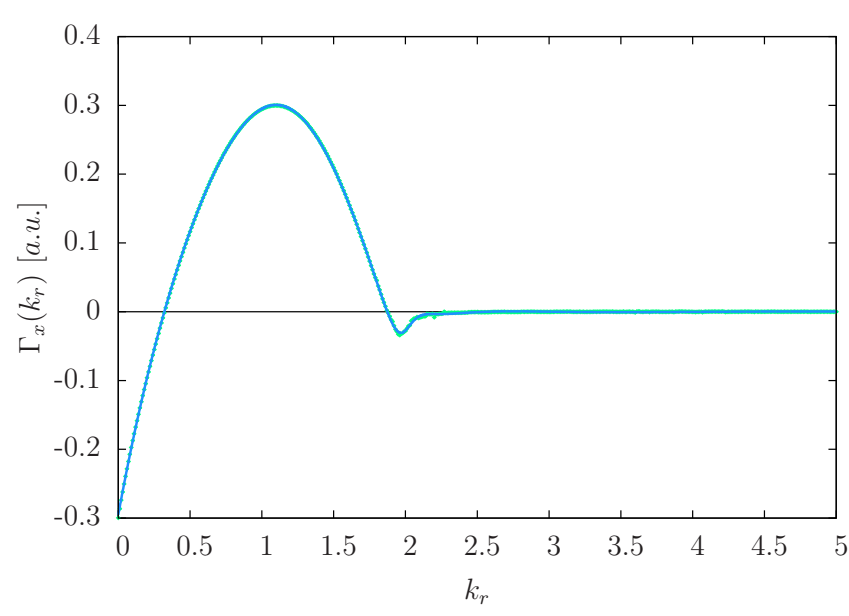

FIG. 2. $\Gamma(k)$ of Eq. (43) and smooth fit.
Let us consider a uniform gas of noninteracting electrons subject to an infinite potential barrier perpendicular to the $x$ axis $(V \rightarrow \infty$ for $x<0$ ). The one-particle density matrix is given by $[71,72]$

$$
\gamma_{1}\left(\mathbf{r}, \mathbf{r}^{\prime}\right)=\bar{n}\left[J\left(u_{f}\right)-J\left(\sqrt{u_{f}^{2}+4 x_{f} x_{f}^{\prime}}\right)\right] \Theta(x) \Theta\left(x^{\prime}\right),
$$

where $\Theta(x)$ is a step function, with $\Theta(x)=1$ for $x>0$ and $\Theta(x)=0$ for $x \leqslant 0$. Here $\bar{n}$ is the average bulk valence electron density, $x_{f}=x k_{f}, x_{f}^{\prime}=x^{\prime} k_{f}, u_{f}=\left|\mathbf{r}-\mathbf{r}^{\prime}\right| k_{f}$, and

$$
J(\xi)=3 j_{1}(\xi) / \xi
$$

with $j_{1}(\xi)=\sin (\xi) / \xi^{2}-\cos (\xi) / \xi$ being the first-order spherical Bessel function. The electron density can be obtained from the single-particle density matrix by taking $u=\left|\mathbf{r}^{\prime}-\mathbf{r}\right|=0$ in Eq. (37). This yields

$$
n(x)=\bar{n}\left[1-J\left(2 x_{f}\right)\right] \Theta(x) .
$$

The WVA for the surface exchange energy density is given by [52]

$$
\gamma_{x}(k)=\int_{0}^{\infty} d u 8 k_{f} u^{2} b_{x}(u) \frac{\sin (k u)}{k u}
$$

where

$$
b_{x}(u)=\int_{-\infty}^{\infty} d x n(x)\left[\rho_{x}(x, u)-\rho_{x}^{\mathrm{unif}}(u)\right] .
$$

The exchange hole $\rho_{x}(x, u)$ of IBM can be obtained from the one-particle density matrix of Eq. (37). With some algebra, we can express the WVA surface exchange energy as [73]

$$
\sigma_{x}=\frac{1}{2} \int_{0}^{\infty} d k_{r} \gamma_{x}\left(k_{r}\right)
$$

where $k_{r}=k / k_{F}, \gamma_{x}\left(k_{r}\right)$ is given by

$$
\begin{aligned}
\gamma_{x}\left(k_{r}\right) & =\frac{8}{k_{f}^{2}} \int_{0}^{\infty} d u_{f} b\left(u_{f}\right) u_{f}^{2} \operatorname{sinc}\left(k_{r} u_{f}\right) \\
& =\frac{1}{\left(\pi r_{s}\right)^{3}} \Gamma\left(k_{r}\right),
\end{aligned}
$$

and

$$
b_{x}\left(u_{f}\right)=-\frac{\bar{n}^{2}}{2 k_{f}} \int_{0}^{\infty} d x_{f} i_{x}\left(x_{f}, u_{f}\right) .
$$

Here $\operatorname{sinc}(x)=\sin (x) / x$, and $i_{x}\left(x_{f}, u_{f}\right)=\sum_{l=1}^{6} \chi_{l}\left(x_{f}, u_{f}\right)$, with $\chi_{l}\left(x_{f}, u_{f}\right)$ being defined by Eq. (3.18) of Ref. [73].

Figure 2 shows the exact variation of $\Gamma\left(k_{r}\right)$ with $k_{r}$. Figure 3 shows the comparison of approximate $\Gamma\left(k_{r}\right)$ with the exact curve (red) for different $m$ values. The area under the curve is proportional to the surface exchange energy. From the electron density and density matrix of IBM given by Eqs. (37) and (39), the exact surface exchange energy can be calculated with the WVA of Eq. (42). Langreth and Perdew [73] reported that the value of $\sigma_{x} 10^{3} r_{s}^{3}$ is 4.0 a.u., where $r_{s}$ is the Seitz radius. This value is slightly smaller than the value obtained earlier by Harris and Jones [74] and Ma and Sahni [75] (4.1 a.u.). Our present work gives 3.99 a.u., which is closer to that of Langreth and Perdew. 


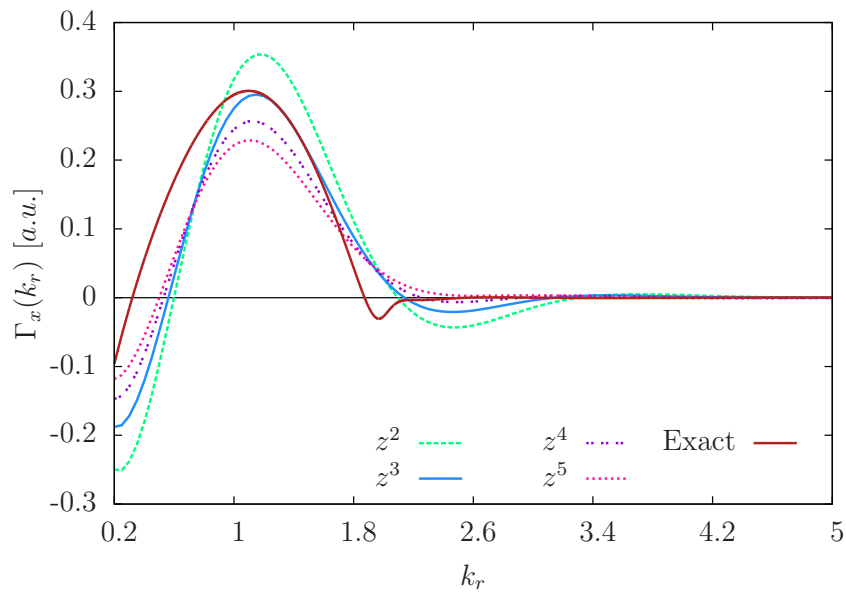

FIG. 3. Analysis of $z$ dependence of the WVA for the present TPSS hole of Eq. (28). The $z^{3}$ curve provides the best fit to the peak region of the exact $\Gamma\left(k_{r}\right)$ of Eq. (43).

\section{D. $z$ dependence of $\mathrm{H}(s, z)$}

The $z$ dependence of $H(s, z)$ [Eq. (36)] can be determined by fitting the TPSS hole to the wave-vector analysis. We start with the specific expressions for the local ingredients of the hole model in IBM.

From the electron density of Eq. (39), the reduced density gradient can be explicitly expressed as

$$
s\left(x_{f}\right)=\frac{3}{2 x_{f}} \frac{\left|\operatorname{sinc}\left(2 x_{f}\right)-J\left(2 x_{f}\right)\right|}{\left[1-J\left(2 x_{f}\right)\right]^{4 / 3}} .
$$

The kinetic energy density can be obtained from the singleparticle density matrix of Eq. (37). This yields

$$
\tau\left(x_{f}\right)=k_{f}^{2} \bar{n}\left\{\frac{3}{10}+\frac{1}{2} J\left(2 x_{f}\right)+\frac{9}{4 x_{f}^{2}}\left[\operatorname{sinc}\left(2 x_{f}\right)-J\left(2 x_{f}\right)\right]\right\} .
$$

Finally, the von Weizsäcker kinetic energy density can be expressed as

$$
\tau_{W}=\frac{9 k_{f}^{2} \bar{n}}{8 x_{f}^{2}}\left\{\frac{\left[\operatorname{sinc}\left(2 x_{f}\right)-J\left(2 x_{f}\right)\right]^{2}}{1-J\left(2 x_{f}\right)}\right\} .
$$

Next, we calculate $\gamma_{x}$ from the TPSS hole. Inserting the TPSS model hole into Eq. (41) yields

$$
\begin{aligned}
b_{x}(u)= & \int_{0}^{\infty} d x n(x)\left[\rho_{\mathrm{x}}^{\mathrm{TPSS}}(x, u)-\rho_{\mathrm{x}}^{u n i f}(u)\right] \\
= & \frac{\tilde{n}^{2}}{k_{f}} \int_{0}^{\infty} d x_{f}\left[1-J\left(2 x_{f}\right)\right]\left\{\left[1-J\left(2 x_{f}\right)\right]\right. \\
& \times J^{\mathrm{TPSS}}\left(u_{f} \sqrt[3]{1-J\left(2 x_{f}\right)}, s\left(x_{f}\right), z\left(x_{f}\right)\right) \\
& \left.-J^{\mathrm{unif}}\left(u_{f}\right)\right\} .
\end{aligned}
$$

[Note that $\Theta(x)$ is implicit on the electron density.] Substituting Eq. (48) into Eq. (40), we obtain

$$
\gamma_{x}(k)=\frac{8 \bar{n}}{3 \pi^{2}} \int_{0}^{\infty} d x_{f} \int_{0}^{\infty} d u_{f} j_{x}\left(u_{f}, x_{f}, k_{r}\right),
$$

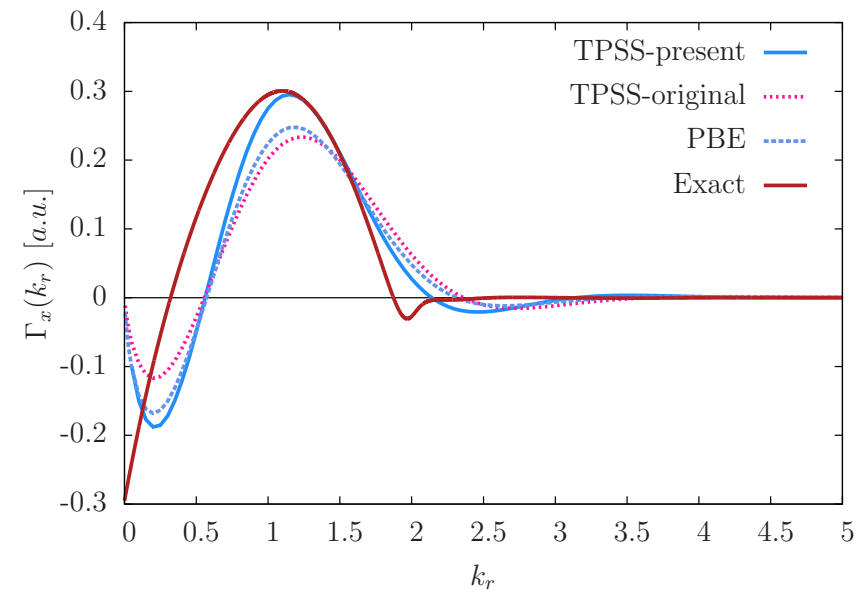

FIG. 4. Comparison of the WVA for the present and original TPSS hole models as well as the PBE hole with the exact one. "TPSS-present" represents the present TPSS hole model, while "TPSS-original" represents the original TPSS model.

where

$$
\begin{aligned}
j_{x}\left(u_{f}, x_{f}, k_{r}\right)= & {\left[\varrho^{2}\left(x_{f}\right) J^{\mathrm{TPSS}}\left(u_{f} \sqrt[3]{\varrho\left(x_{f}\right)}, s\left(x_{f}\right), z\left(x_{f}\right)\right)\right.} \\
& \left.-\varrho\left(x_{f}\right) J^{\mathrm{unif}}\left(u_{f}\right)\right] \operatorname{sinc}\left(k_{r} u_{f}\right) u_{f}^{2}
\end{aligned}
$$

and $\varrho\left(x_{f}\right)=1-J\left(2 x_{f}\right)$. Rearrangement of Eq. (49) leads to the final expression

$$
\gamma_{x}(k)=\frac{1}{\left(\pi r_{s}\right)^{3}} \Gamma^{\mathrm{TPSS}}\left(k_{r}\right)
$$

where

$$
\Gamma^{\mathrm{TPSS}}\left(k_{r}\right)=2 \int_{0}^{\infty} \int_{0}^{\infty} d x_{f} d u_{f} j_{x}\left(u_{f}, x_{f}, k_{r}\right) .
$$

Figure 3 shows the comparison of $H(s, z)$ with different choices of $m$ to the exact one. From Fig. 3, we see that the best fit to the exact $\Gamma\left(k_{r}\right)$ in the peak region is $m=3$. Figure 4 shows that, compared to the WVA of the LSDA, PBE, and original TPSS holes, the WVA of the present model is closest to the exact one in the peak region. To further understand the original and present TPSS models, we plot the TPSS shape function of the present and the original models in IBM at $z=0.55$, as shown by Figs. 5 and 6 , respectively. From Figs. 5 and 6 , we observe that while the present model hole is always negative, the original TPSS hole can be positive in some range of $u_{f}$ and $s$.

To check our wave-vector analysis for the surface exchange energy, we have computed $\sigma_{x}$ from

$$
\sigma_{x}=\int_{-\infty}^{\infty} d x n(x)\left[\epsilon_{x}(n)-\epsilon_{x}^{\mathrm{unif}}(\bar{n})\right] .
$$

The results are shown in Table II. From Table II, we can see that the surface energy from the WVA of the TPSS hole (both original and the present version) agrees very well with the surface energy calculated directly from the TPSS exchange functional [Eq. (53)]. Furthermore, the TPSS surface energy is closer to the exact value than those of the LSDA and PBE. The LSDA significantly overestimates the surface exchange energy, while the PBE gives underestimation. These 


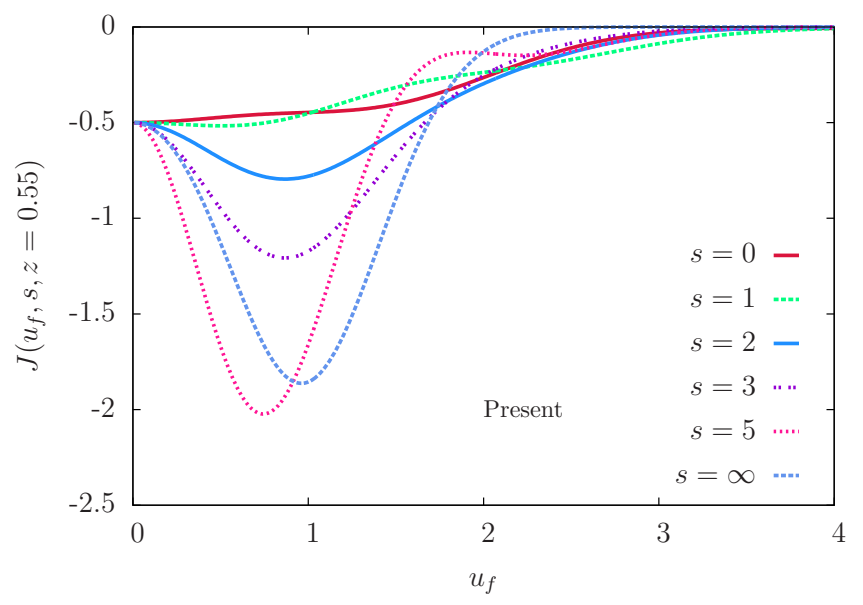

FIG. 5. Present TPSS shape function of Eq. (28) for $z=0.55$.

observations are consistent with those evaluated from the jellium surface linear potential model [45]. It is interesting to note that even though the original TPSS shape function in a certain range is positive, the surface energy from the original TPSS hole is the same as that from the present model. This result is simply due to the cancellation of the original hole model between positive values and too negative values at certain $u_{f}$ and $s$ values, as seen from the comparison of Fig. 6 to Fig. 5. The IBM surface energy presents a great challenge to semilocal DFT. It is more difficult to get it right than the surface energy of the jellium model with finite linear potential because the electron density at the surface of IBM is highly inhomogeneous due to the sharp cutoff at surface and is too far from the slowly varying regime where semilocal DFT can be exact (e.g., TPSS functional).

Figure 7 shows a comparison of the differences of the system-averaged hole between the approximations and the exact curve for the LSDA, PBE, and the original and present TPSS exchange hole models of the Ne atom, in which $z$ is, in general, different from 0 (slowly varying density) and 1 (iso-orbital density). The PBE and LSDA curves are plotted with the hole models of Ref. [67]. From Fig. 7 we can see that, except for the small region near the core, the present

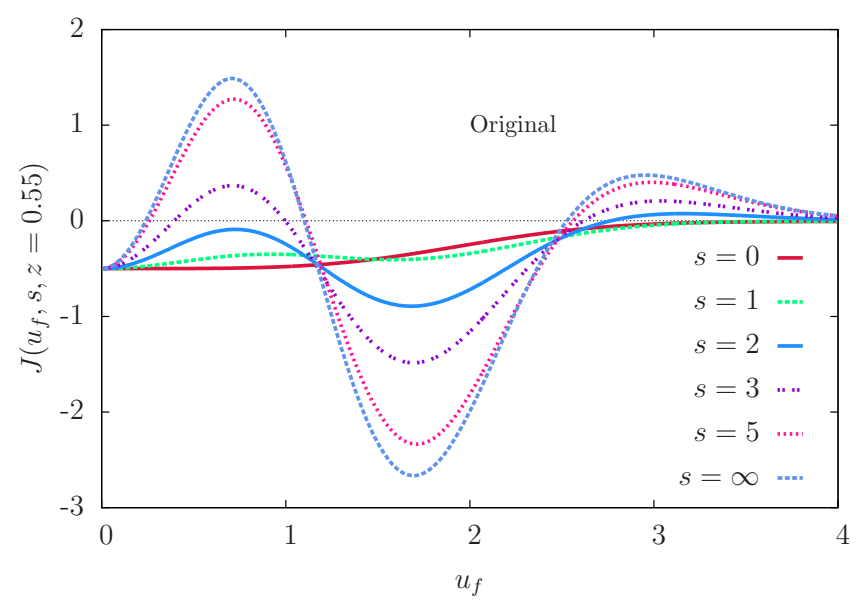

FIG. 6. Original TPSS shape function for $z=0.55$.
TABLE II. Comparison of the surface exchange energies (in a.u.) of the IBM surface (expressed as $\sigma_{x} r_{s}{ }^{3} 10^{3}$ ) calculated directly with exchange energy functionals and with the WVA formula. The exact value (obtained in this work) is 3.99 a.u.

\begin{tabular}{lcc}
\hline \hline & Eq. (53) & WVA integration \\
\hline LSDA & 6.318 & \\
PBE & 2.576 & \\
TPSS & 2.945 & 2.95 (original hole) \\
& & 2.95 (present hole) \\
\hline
\end{tabular}

TPSS hole model is closer to the exact one than the original TPSS hole model, but both TPSS models obviously improve the system-averaged holes of the LSDA and PBE.

\section{TPSS HOLE IN THE GAUGE OF THE CONVENTIONAL EXACT EXCHANGE}

The shape function explicitly depends on the enhancement factor via the energy constraint of Eq. (17). The latter may be altered by adding an arbitrary amount of the Laplacian of the density without changing the total exchange energy. This ambiguity of the exchange energy density [66] leads to the ambiguity of the semilocal exchange hole. Our primary goal of this work is to develop a semilocal exchange hole in the gauge of the conventional exact exchange. This is partly motivated by the fact that, in the development of range-separated density functionals, the exact exchange part is usually provided in the conventional gauge.

The exact exchange energy density in the conventional gauge can be conveniently evaluated with the Della SalaGörling (DSG) [76] identity resolution

$$
e_{\mathrm{conv}}^{x}(\mathbf{r})=\frac{1}{2} \sum_{\mu \nu} Q_{\mu \nu}^{\sigma} \chi_{\mu}(\mathbf{r}) \chi_{\nu}^{*}(\mathbf{r}),
$$

where $Q^{\sigma}$ is the spin block of the DSG matrix [54]. However, many semilocal exchange energy densities or enhancement

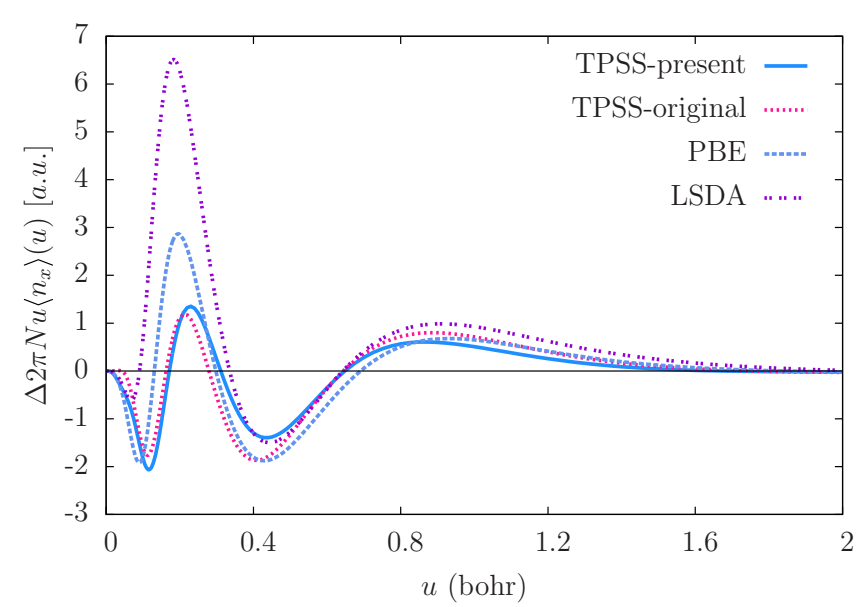

FIG. 7. Comparison of the difference of the system-averaged hole between the approximations and the exact curve for the $\mathrm{Ne}$ atom. "TPSS-present" represents the present TPSS hole model, while "TPSS-original" represents the original TPSS model. 
factors of Eq. (17) are not in the gauge of the conventional exact exchange due to the constraints such as the Lieb-Oxford bound and the slowly varying gradient expansion (with integration by parts) imposed on the enhancement factor. For example, for the two-electron exponential density, the conventionally defined exact enhancement factor is less than 1 near the nucleus, while the TPSS enhancement factor is $F_{\mathrm{x}}^{\mathrm{TPSS}} \geqslant 1$ by design. In the density tail region, the conventional exact enhancement factor tends to infinity, but the maximum value of $F_{\mathrm{x}}^{\mathrm{TPSS}}$ is 1.804 . To construct the TPSS exchange hole in the conventional gauge, we can replace the original energy density constraint [Eq. (17)], which was used in the construction of the original TPSS exchange hole [52], with the TPSS exchange energy density or enhancement factor in the conventional gauge. In this gauge, the TPSS exchange energy density can be written as [54]

$$
e_{\mathrm{x}}^{\mathrm{TPSS}}(\mathbf{r})=e_{\mathrm{x}}^{\mathrm{TPSS}, \text { conv }}(\mathbf{r})+G(\mathbf{r}),
$$

where $e_{\mathrm{x}}^{\text {TPSS }}(\mathbf{r})$ is the standard TPSS exchange energy density [17] (i.e., $\lambda=0.92$ ) and $e_{\mathrm{x}}^{\mathrm{TPS}, \text { conv }}(\mathbf{r})$ is the TPSS exchange energy density in the exact conventional gauge (i.e., $\lambda=1$ ), with $\lambda$ being the general coordinate transformation parameter $[54,64,65]$. Here $e_{x}(\mathbf{r})=n(\mathbf{r}) \epsilon_{x}(\mathbf{r})$. Equivalently, we can also write

$$
e_{\mathrm{x}}^{\mathrm{ex}, \mathrm{tpssg}}(\mathbf{r})=e_{\mathrm{x}}^{\mathrm{ex}, \mathrm{conv}}(\mathbf{r})+G(\mathbf{r}),
$$

where $e_{\mathrm{x}}^{\text {ex,tpssg }}(\mathbf{r})$ is the exact exchange energy density in TPSS gauge and $e_{\mathrm{x}}^{\text {ex,conv }}(\mathbf{r})$ is the exact conventional exchange energy density evaluated from the single-particle density matrix [Eqs. (2)-(6)]. Based on the uniform and nonuniform coordinate scaling properties of the exact exchange energy density, Tao, Staroverov, Scuseria, and Perdew (TSSP) [54] proposed a gauge function

$$
\begin{gathered}
G(\mathbf{r})=a \boldsymbol{\nabla} \cdot[f(\mathbf{r}) \nabla \tilde{\epsilon}], \\
f=\frac{n / \tilde{\epsilon}^{2}}{1+c\left(n / \tilde{\epsilon}^{3}\right)^{2}}\left(\frac{\tau^{W}}{\tau}\right)^{b} .
\end{gathered}
$$

Here $a=0.015$ and $c=0.04$ are determined by a fit to the conventional exact exchange energy density of the $\mathrm{H}$ atom, and $b$ is an integer which is chosen to be 4 due to the consideration of sodium jellium sphere clusters. $\tilde{\epsilon}=-\epsilon_{x}^{\text {ex,conv }}$ is the exact exchange energy density in the conventional gauge. This gauge function is integrated to zero, i.e., $\int d^{3} r G(\mathbf{r})=0$, as required. It satisfies the correct uniform coordinate scaling relation, $G_{\lambda}(\mathbf{r})=\lambda^{4} G(\lambda \mathbf{r})$, and nonuniform coordinate scaling relation $G_{\lambda}^{x}(x, y, z)=\lambda G(\lambda x, y, z)$.

However, in the far density tail $(r \rightarrow \infty)$ of an atom, the exact exchange energy density in the conventional gauge decays as $e_{\mathrm{x}}^{\text {ex,conv }} \sim-n / 2 r$, but the original TSSP gauge function decays as $G(\mathbf{r}) \sim n$. As a result, the exchange energy density in this gauge becomes positive in the density tail region. In order to fix this deficiency, we impose a constraint on the density tail,

$$
\lim _{r \rightarrow \infty} \frac{G}{e_{\mathrm{x}}^{\text {conv }}}=0 .
$$

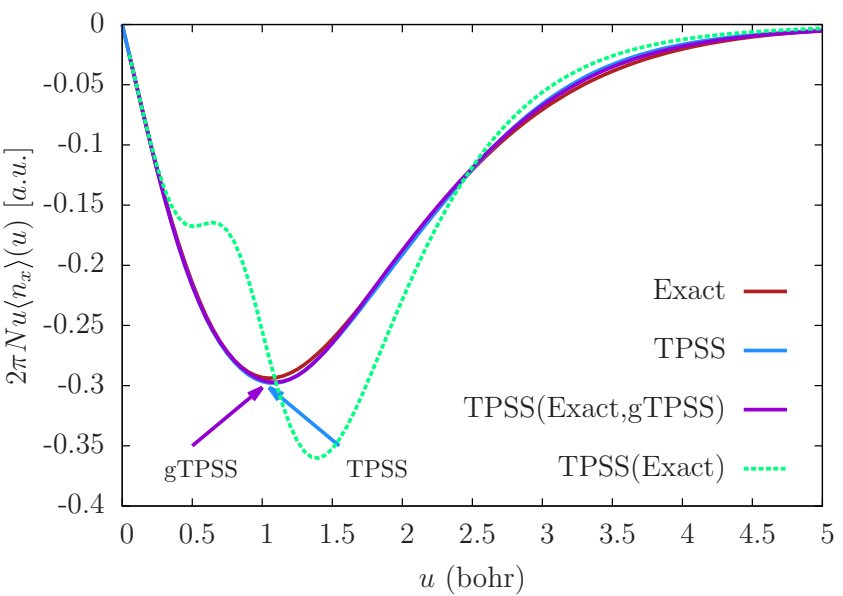

FIG. 8. Comparison of the system-averaged holes for the twoelectron exponential density. "Exact" represents the conventional exact system-averaged hole $\rho_{x}^{\text {exact }}(\mathbf{r}, u)$ (red) from Eqs. (4)-(6), "TPSS" represents the present TPSS system-averaged hole (blue) from Eqs. (28)-(31) and (36) with Table I and $m=3$, "TPSS(Exact)" represents the system-averaged hole (green) generated from the TPSS hole but with $F_{\mathrm{x}}^{\mathrm{TPSS}}(\mathbf{r})$ of Eq. (17) replaced by $F_{\mathrm{x}}^{\text {exact }}(\mathbf{r})$, and "TPSS(Exact,gTPSS)" represents the system-averaged hole generated from $e_{\mathrm{x}}^{\text {ex,tpssg }}(\mathbf{r})$ of Eq. (56) (purple).

This can be achieved by requiring that in the $r \rightarrow \infty$ limit, $G$ decays as $n^{p}$ with $p>1$. Here we choose $p=\frac{3}{2}$ and take the same form of the TSSP gauge function, but with $f$ given by

$$
f=\frac{\left(n / \tilde{\epsilon}^{\frac{7}{3}}\right)^{3 / 2}}{1+c\left(n / \tilde{\epsilon}^{3}\right)^{5 / 2}}\left(\frac{\tau^{W}}{\tau}\right)^{b} .
$$

Here $a=0.01799$ and $c=0.00494$ are determined by fitting the TPSS system-averaged hole in the conventional gauge to the exact system-averaged hole of the two-electron exponential density. The fitting procedure is the same as that in the determination of the $H(s, z=1)$ function. The parameter $b=$ 4 remains the same as that in the original version [Eq. (58)]. our present gauge function retains all the correct properties that the original gauge function satisfies, including the nonuniform coordinate scaling property.

Figure 8 shows the comparison of the present TPSS systemaveraged exchange hole and the exact conventional systemaveraged exchange hole calculated from the present TPSS hole model but with $F_{\mathrm{x}}^{\mathrm{TPSS}}(\mathbf{r})$ of Eq. (17) replaced by $F_{\mathrm{x}}^{\text {exact }}(\mathbf{r})$ with and without the gauge correction of Eq. (60) to the exact conventional one [Eqs. (4)-(6)]. From Fig. 8 we can observe that the exact system-averaged exchange hole generated from the present TPSS hole model without the gauge correction significantly deviates from the exact system-averaged hole. However, the agreement has been significantly improved with our present gauge correction [Eq. (60)].

Figure 9 shows the comparison of the TPSS exchange energy density evaluated with the TPSS functional without and with the gauge correction to the exact conventional exchange energy density for the two-electron exponential density. From Fig. 9, we can observe that the effect of the present gauge correction defined by Eq. (60) is small for the present TPSS hole. However, as observed in Fig. 8, it is important for the 


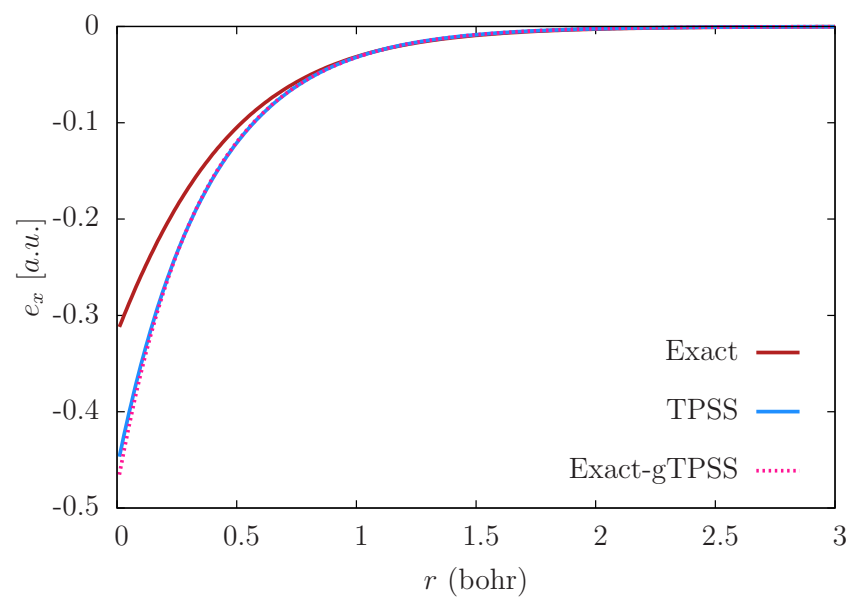

FIG. 9. Comparison of the exchange energy densities for the two-electron exponential density calculated with different approximations to the exact one. "TPSS" represents the TPSS exchange energy density calculated directly from the TPSS exchange energy functional; "gTPSS" represents the gauge-corrected TPSS exchange energy density.

conventional exact exchange hole evaluated with the present TPSS hole.

\section{APPLICATION TO RANGE-SEPARATED EXCHANGE FUNCTIONAL}

As a simple application, we apply the present TPSS hole model to construct a range-separated functional. In general, there are two ways to construct a range-separated functional, simply depending on the need. For example, we may employ a semilocal DFT as the long-range part, while the exact exchange is used for the short-range part, as pioneered by Heyd, Scuseria, and Ernzerhof [14]. This kind of range-separated functional is developed largely for solids and is particularly useful for metallic solids because usual hybrids require much larger momentum cutoff for metallic systems with electrons nonlocalized. Nevertheless, this range-separated functional is also accurate for molecules. We may also employ a semilocal DFT for the short-range part, while the exact exchange is used for the long-range part, as developed by Henderson et al. [67] on the basis of the PBE hole. These kinds of range-separated functionals are usually developed for molecular calculations because the improved long-range part of the exchange hole will improve the description of molecular properties. Many range-separated functionals have been proposed [48,77-81]. In the following, we will explore the TPSS hole-based rangeseparated functional with the TPSS exchange functional being the long-range (LR) part and the Hartree-Fock exchange being the short-range (SR) part, aiming to improve the too small band gaps and reaction barrier heights of the TPSS functional.

The idea of the construction of our TPSS-based rangeseparated functional is rooted in the construction of the usual one-parameter hybrid functionals, which, in general, can be written as

$$
E_{x c}^{\mathrm{hybrid}}=a E_{\mathrm{x}}^{\mathrm{HF}}+(1-a) E_{\mathrm{x}}^{\mathrm{sl}}+E_{\mathrm{c}}^{\mathrm{sl}},
$$

TABLE III. Band gaps (in eV) calculated with the LSDA, PBE, HSE, TPSS, and TPSS-based range-separated functional with $a=$ 0.25 and $\omega=0.10$ ( $\mathrm{PW}=$ present work) compared to experiments. ME stands for mean error and MAE stands for mean absolute error.

\begin{tabular}{lcccccc}
\hline \hline & LSDA & PBE & TPSS & HSE & PW & Expt. \\
\hline $\mathrm{C}$ & 4.17 & 4.2 & 4.24 & 5.43 & 5.48 & 5.48 \\
$\mathrm{CdSe}$ & 0.31 & 0.63 & 0.85 & 1.48 & 1.82 & 1.90 \\
$\mathrm{GaAs}$ & 0.04 & 0.36 & 0.6 & 1.11 & 1.44 & 1.52 \\
$\mathrm{GaN}$ & 2.15 & 2.22 & 2.18 & 3.48 & 3.5 & 3.50 \\
$\mathrm{GaP}$ & 1.56 & 1.74 & 1.83 & 2.39 & 2.53 & 2.35 \\
$\mathrm{Ge}$ & & 0.13 & 0.32 & 0.8 & 0.99 & 0.74 \\
$\mathrm{InAs}$ & 0 & & 0.08 & 0.57 & 0.85 & 0.41 \\
$\mathrm{InN}$ & 0 & 0 & 0 & 0.72 & 0.75 & 0.69 \\
$\mathrm{InSb}$ & & 0 & & 0.47 & 0.73 & 0.23 \\
$\mathrm{Si}$ & 0.53 & 0.62 & 0.71 & 1.2 & 1.31 & 1.17 \\
$\mathrm{ZnS}$ & 2.02 & 2.3 & 2.53 & 3.44 & 3.78 & 3.66 \\
$\mathrm{ME}$ & -0.89 & -0.86 & -0.76 & -0.12 & 0.14 & \\
$\mathrm{MAE}$ & 0.89 & 0.86 & 0.76 & 0.15 & 0.17 & \\
\hline \hline
\end{tabular}

where $a$ is the mixing parameter that controls the amount of exact exchange mixed into a semilocal (sl) functional.

Following the prescription of Heyd, Scuseria, and Ernzerhof (HSE) [14], we write the TPSS-based range-separated functional as

$$
E_{\mathrm{xc}}=a E_{\mathrm{x}}^{\mathrm{HF}, \mathrm{SR}}+(1-a) E_{\mathrm{x}}^{\mathrm{sl}, \mathrm{SR}}+E_{\mathrm{x}}^{\mathrm{sl}, \mathrm{LR}}+E_{\mathrm{c}}^{\mathrm{sl}},
$$

where $E_{\mathrm{x}}^{\mathrm{HF}, \mathrm{SR}}$ is the Hartree-Fock (HF) exchange serving as part of the short-range contribution, while $E_{\mathrm{x}}^{\mathrm{sl}, \mathrm{SR}}$ is the TPSS exchange that provides the rest of the short-range contribution. $E_{\mathrm{c}}^{\mathrm{sl}}$ is the TPSS correlation. The long-range contribution is provided fully by the TPSS exchange $E_{\mathrm{x}}^{\mathrm{sl}, \mathrm{LR}}$. They are given, respectively, by

$$
\begin{aligned}
& \epsilon_{\mathrm{x}}^{\mathrm{HF}, \mathrm{SR}}=\frac{1}{2} \int_{0}^{\infty} d u 4 \pi u^{2} \rho_{\mathrm{x}}^{\mathrm{HF}}(\mathbf{r}, u) \frac{\operatorname{erfc}(\omega u)}{u}, \\
& \epsilon_{\mathrm{x}}^{\mathrm{sl}, \mathrm{SR}}=\frac{1}{2} \int_{0}^{\infty} d u 4 \pi u^{2} \rho_{\mathrm{x}}^{\mathrm{TPSS}}(\mathbf{r}, u) \frac{\operatorname{erfc}(\omega u)}{u}, \\
& \epsilon_{\mathrm{x}}^{\mathrm{sl}, \mathrm{LR}}=\frac{1}{2} \int_{0}^{\infty} d u 4 \pi u^{2} \rho_{\mathrm{x}}^{\mathrm{TPSS}}(\mathbf{r}, u) \frac{\operatorname{erf}(\omega u)}{u},
\end{aligned}
$$

where $\omega$ is a range-separation parameter and $\operatorname{erf}(x)$ is the error function defined by Eq. (27). From Eqs. (62)-(65), we can see that the amount of exact exchange mixing is controlled by two parameters, $a$ and $\omega$. Determination of them is discussed below. To test this functional, we have implemented it into the developmental version of GAUSSIAN 09 [82].

In the TPSS-based hybrid functional (TPSSh) [33], $a=0.1$ was fitted to $223 \mathrm{G} 3 / 99$ atomization energies. In other words, the optimal value of $a$ is 0.1 for TPSSh. If we consider only atomization energy, then the best value of $\omega$ in the TPSS-based range-separated functional should be zero if $a=0.1$ is chosen. Since, in the range-separated functional, some amount of the exact exchange (here the long-range part) in the TPSSh is replaced by the TPSS functional, to compensate for this, we need a value of $a$ larger than 0.1 . Then we can find the best range-separated parameter $\omega$ by fitting to some electronic properties. This situation is different from PBE-based range-separated functionals, in which the mixing 
TABLE IV. AE6 atomization energies (in $\mathrm{kcal} / \mathrm{mol}$ ) calculated with the LSDA, PBE, TPSS, TPSSh, HSE, and TPSS-based rangeseparated functional with $a=0.25$ and $\omega=0.10$ (PW $=$ present work) compared to experimental values [84]. ME stands for mean error and MAE stands for mean absolute error.

\begin{tabular}{lcrrcccc}
\hline \hline & LSDA & PBE & TPSS & TPSSh & HSE & PW & Expt. \\
\hline $\mathrm{SiH}_{4}$ & 347.4 & 313.2 & 333.7 & 333.6 & 314.5 & 333.6 & 322.4 \\
$\mathrm{SiO}$ & 223.9 & 195.7 & 186.7 & 182.0 & 182.1 & 175.4 & 192.1 \\
$\mathrm{~S}_{2}$ & 135.1 & 114.8 & 108.7 & 105.9 & 106.3 & 101.9 & 101.7 \\
$\mathrm{C}_{3} \mathrm{H}_{4}$ & 802.1 & 721.2 & 707.5 & 704.4 & 705.9 & 699.9 & 704.8 \\
$\mathrm{C}_{2} \mathrm{H}_{2} \mathrm{O}_{2}$ & 754.9 & 665.1 & 636.0 & 628.0 & 635.3 & 616.4 & 633.4 \\
$\mathrm{C}_{4} \mathrm{H}_{8}$ & 1304 & 1168 & 1156 & 1154 & 1152 & 1152 & 1149 \\
$\mathrm{ME}$ & 77.4 & 12.4 & 4.1 & 0.75 & -1.2 & -4.0 & \\
$\mathrm{MAE}$ & 77.4 & 15.5 & 5.9 & 6.1 & 4.8 & 8.8 & \\
\hline \hline
\end{tabular}

parameter $a=1 / 4$ in PBE0 [13] can be retained. To avoid possible overfitting, here we choose $a=1 / 4$, a value that was recommended by Perdew, Ernzerhof, and Burke [83] and adopted with the PBE0 functional [13]. The parameter $\omega$ is determined by a fit to the band gap of diamond (C). This yields $\omega=0.1$. Then we apply this range-separated functional to calculate the band gaps of 10 semiconductors. The results are listed in Table III. From Table III, we see that the band gaps of this range-separated functional are remarkably accurate, with a mean absolute deviation from experiments of only $0.17 \mathrm{eV}$, about the same accuracy as the HSE functional. We can also see from Table III that the TPSS-based range-separated functional will be expected to yield a more accurate description for large band-gap materials and therefore provides an alternative choice for band-gap and other solid-state calculations.

Next, we apply our range-separated functional to calculate atomization energies of six molecules (AE6). The results are listed in Table IV. From Table IV, we can see that our rangeseparated functional worsens the atomization energies of the TPSS functional for this special set only by about $3 \mathrm{kcal} / \mathrm{mol}$. This error is still smaller than many other DFT methods such as the LSDA and PBE.

Reaction barrier heights are a decisive quantity in the study of chemical kinetics. However, semilocal functionals tend to underestimate this quantity. As another application, we apply our range-separated functional to calculate six representative reaction barrier heights (BH6), which consist of three forward (f) and three reverse (r) barrier heights. The results are listed in Table V. For comparison, we also calculated these barrier heights using the PBE, TPSS, TPSSh, and HSE. From Table V, we observe that our range-separated functional provides a substantially improved description for barrier heights compared to the TPSS and TPSSh functionals.

\section{CONCLUSION}

In conclusion, we have developed a conventional semilocal exchange hole underlying the TPSS exchange functional. The hole is exact in the uniform-gas limit and accurate for compact iso-orbital densities. It satisfies the constraints that the TPSS exchange functional satisfies. It also satisfies the constraints on the conventional exchange hole. The hole can be regarded as an interpolation between the two-electron exponential density and the IBM jellium surface. Numerical tests on $\mathrm{H}$ and $\mathrm{Ne}$ atoms show that the hole mimics the conventional exact exchange hole quite accurately. In particular, with our present gauge function correction, the hole model can generate the exact system-averaged hole accurately.

As an immediate application, we have employed the exchange hole model to construct a range-separated functional. Our tests show that this functional can yield accurate band gaps, in particular for insulators, and reaction barrier heights without losing much accuracy for atomization energies. Since TPSS is more accurate than PBE for many properties and since the PBE hole has been thoroughly explored in recent years, development of TPSS hole-based range-separated functionals is of general interest. Recently, Arbuznikov and Kaupp [21] found that the gauge function has some effect on local hybrid functionals. It is expected that our present gauge function can be useful in the development of nonlocal functionals.

\section{ACKNOWLEDGMENTS}

We thank T. M. Henderson for providing the code for the exchange hole of the $\mathrm{Ne}$ atom. J.T. acknowledges support from the NSF under Grant No. CHE 1640584. J.T. also acknowledges support from Temple start-up via John P. Perdew. I.W.B. and G.E.S. were supported by the U.S. Department of Energy, Office of Basic Energy Sciences, Computational and Theoretical Chemistry Program under Award No. DE-FG0209ER16053. G.E.S. is a Welch Foundation Chair (C-0036).

TABLE V. BH6 reaction barrier heights (in kcal/mol) calculated with the PBE, TPSS, TPSSh, HSE, and TPSS-based range-separated functional with $a=0.25$ and $\omega=0.10$ (PW = present work) in comparison with reference values [85,86]. Here f (r) = forward (reverse) barrier height. ME stands for mean error and MAE stands for mean absolute error.

\begin{tabular}{|c|c|c|c|c|c|c|}
\hline & PBE & TPSS & TPSSh & HSE & PW & Reference \\
\hline \multirow{2}{*}{$\mathrm{OH}+\mathrm{CH}_{4} \rightarrow \mathrm{CH}_{3}+\mathrm{H}_{2} \mathrm{O}$} & -5.29 & -0.97 & 1.50 & 1.96 & 4.86 & $6.54(f)$ \\
\hline & 8.95 & 9.90 & 11.79 & 13.9 & 14.3 & $19.6(r)$ \\
\hline \multirow[t]{2}{*}{$\mathrm{H}+\mathrm{OH} \rightarrow \mathrm{O}+\mathrm{H}_{2}$} & 3.69 & -1.56 & -0.15 & 7.06 & 1.75 & $10.5(\mathrm{f})$ \\
\hline & -1.47 & 4.73 & 6.90 & 5.93 & 9.89 & $12.9(\mathrm{r})$ \\
\hline \multirow[t]{2}{*}{$\mathrm{H}+\mathrm{H}_{2} \mathrm{~S} \rightarrow \mathrm{H}_{2}+\mathrm{HS}$} & -1.20 & -4.55 & -3.72 & 1.03 & -2.64 & $3.55(\mathrm{f})$ \\
\hline & 9.40 & 12.72 & 13.4 & 12.4 & 14.4 & $17.3(\mathrm{r})$ \\
\hline ME & -9.37 & -8.34 & -6.76 & -4.66 & -4.63 & \\
\hline MAE & 9.37 & 8.34 & 6.76 & 4.66 & 4.63 & \\
\hline
\end{tabular}


[1] W. Kohn and L. J. Sham, Phys. Rev. 140, A1133 (1965).

[2] R. G. Parr and W. Yang, Density Functional Theory of Atoms and Molecules (Oxford University Press, New York, 1989).

[3] R. M. Dreizler and E. K. U. Gross, Density Functional Theory (Springer, Berlin, 1990).

[4] J. P. Perdew and Y. Wang, Phys. Rev. B 33, 8800 (1986).

[5] A. D. Becke, Phys. Rev. A 38, 3098 (1988).

[6] C. Lee, W. Yang, and R. G. Parr, Phys. Rev. B 37, 785 (1988).

[7] A. D. Becke and M. R. Roussel, Phys. Rev. A 39, 3761 (1989).

[8] A. D. Becke, J. Chem. Phys. 104, 1040 (1996).

[9] P. J. Stephens, F. J. Devlin, C. F. Chabalowski, and M. J. Frisch, J. Phys. Chem. 98, 11623 (1994).

[10] J. P. Perdew, K. Burke, and M. Ernzerhof, Phys. Rev. Lett. 77, 3865 (1996).

[11] T. V. Voorhis and G. E. Scuseria, J. Chem. Phys. 109, 400 (1998).

[12] F. A. Hamprecht, A. J. Cohen, D. J. Tozer, and N. C. Handy, J. Chem. Phys. 109, 6264 (1998).

[13] M. Ernzerhof and G. E. Scuseria, J. Chem. Phys. 110, 5029 (1999).

[14] J. Heyd, G. E. Scuseria, and M. Ernzerhof, J. Chem. Phys. 118, 8207 (2003).

[15] R. Armiento and A. E. Mattsson, Phys. Rev. B 72, 085108 (2005).

[16] Y. Zhao and D. G. Truhlar, J. Chem. Phys. 125, 194101 (2006).

[17] J. Tao, J. P. Perdew, V. N. Staroverov, and G. E. Scuseria, Phys. Rev. Lett. 91, 146401 (2003).

[18] J. P. Perdew, A. Ruzsinszky, G. I. Csonka, L. A. Constantin, and J. Sun, Phys. Rev. Lett. 103, 026403 (2009).

[19] J. P. Perdew, A. Ruzsinszky, G. I. Csonka, O. A. Vydrov, G. E. Scuseria, L. A. Constantin, X. Zhou, and K. Burke, Phys. Rev. Lett. 100, 136406 (2008).

[20] J. Sun, A. Ruzsinszky, and J. P. Perdew, Phys. Rev. Lett. 115, 036402 (2015).

[21] A. V. Arbuznikov and M. Kaupp, J. Chem. Phys. 141, 204101 (2014).

[22] L. A. Constantin, A. Terentjevs, F. Della Sala, P. Cortona, and E. Fabiano, Phys. Rev. B 93, 045126 (2016).

[23] J. C. Pacheco-Kato, J. M. del Campo, J. L. Gázquez, S. B. Trickey, and A. Vela, Chem. Phys. Lett. 651, 268 (2016).

[24] J. Tao and Y. Mo, Phys. Rev. Lett. 117, 073001 (2016).

[25] S. H. Vosko, L. Wilk, and M. Nusair, Can. J. Phys. 58, 1200 (1980).

[26] J. P. Perdew and Y. Wang, Phys. Rev. B 45, 13244 (1992).

[27] J. P. Perdew, J. A. Chevary, S. H. Vosko, K. A. Jackson, M. R. Pederson, D. J. Singh, and C. Fiolhais, Phys. Rev. B 46, 6671 (1992).

[28] Z. Wu and R. E. Cohen, Phys. Rev. B 73, 235116 (2006).

[29] C. J. Cramer and D. G. Truhlar, Phys. Chem. Chem. Phys. 11, 10757 (2009).

[30] R. Peverati and D. G. Truhlar, Philos. Trans. R. Soc. A 372, 20120476 (2011).

[31] A. J. Cohen, P. Mori-Sanchez, and W. Yang, Chem. Rev. 112, 289 (2012).

[32] A. D. Becke, J. Chem. Phys. 140, 18A301 (2014).

[33] V. N. Staroverov, G. E. Scuseria, J. Tao, and J. P. Perdew, J. Chem. Phys. 119, 12129 (2003); 121, 11507(E) (2004).

[34] P. Hao, J. Sun, B. Xiao, A. Ruzsinszky, G. I. Csonka, J. Tao, S. Glindmeyer, and J. P. Perdew, J. Chem. Theory Comput. 9, 355 (2013).
[35] L. Goerigk and S. Grimme, J. Chem. Theory Comput. 6, 107 (2010).

[36] L. Goerigk and S. Grimme, J. Chem. Theory Comput. 7, 291 (2011).

[37] Y. Mo, G. Tian, R. Car, V. N. Staroverov, G. E. Scuseria, and J. Tao, J. Chem. Phys. 145, 234306 (2016).

[38] Y. Mo, G. Tian, and J. Tao, arXiv:1610.00643.

[39] D. Jacquemin and C. Adamo, J. Chem. Theory Comput. 7, 369 (2011).

[40] Y. Mo, G. Tian, and J. Tao, arXiv:1610.00775.

[41] G. I. Csonka, J. P. Perdew, A. Ruzsinszky, P. H. T. Philipsen, S. Lebégue, J. Paier, O. A. Vydrov, and J. G. Ángyán, Phys. Rev. B 79, 155107 (2009).

[42] P. Haas, F. Tran, and P. Blaha, Phys. Rev. B 79, 085104 (2009).

[43] F. Tran, J. Stelzl, and P. Blaha, J. Chem. Phys. 144, 204120 (2016).

[44] Y. Mo, R. Car, V. N. Staroverov, G. E. Scuseria, and J. Tao, Phys. Rev. B 95, 035118 (2017).

[45] V. N. Staroverov, G. E. Scuseria, J. Tao, and J. P. Perdew, Phys. Rev. B 69, 075102 (2004).

[46] J. P. Perdew, V. N. Staroverov, J. Tao, and G. E. Scuseria, Phys. Rev. A 78, 052513 (2008).

[47] J. Jaramillo, G. E. Scuseria, and M. Ernzerhof, J. Chem. Phys. 118, 1068 (2003).

[48] R. Peverati and D. G. Truhlar, Phys. Chem. Lett. 2, 2810 (2011).

[49] J. P. Perdew, K. Burke, and Y. Wang, Phys. Rev. B 54, 16533 (1996).

[50] J. Tao, Density Functional Theory of Atoms, Molecules, and Solids (VDM, Saarbrücken, Germany, 2010).

[51] M. Ernzerhof and J. P. Perdew, J. Chem. Phys. 109, 3313 (1998).

[52] L. A. Constantin, J. P. Perdew, and J. Tao, Phys. Rev. B 73, 205104 (2006).

[53] L. A. Constantin, E. Fabiano, and F. Della Sala, Phys. Rev. B 88, 125112 (2013).

[54] J. Tao, V. N. Staroverov, G. E. Scuseria, and J. P. Perdew, Phys. Rev. A 77, 012509 (2008).

[55] S. Refaely-Abramson, R. Baer, and L. Kronik, Phys. Rev. B 84, 075144 (2011).

[56] R. Baer, E. Livshits, and U. Salzner, Annu. Rev. Phys. Chem. 61, 85 (2010).

[57] M. Modrzejewski, M. Hapka, G. Chalasinski, and M. M. Szczesniak, J. Chem. Theory Comput. 12, 3662 (2016).

[58] L. Schimka, J. Harl, and G. Kresse, J. Chem. Phys. 134, 024116 (2011).

[59] A. E. Mattsson and W. Kohn, J. Chem. Phys. 115, 3441 (2001).

[60] R. Armiento and A. E. Mattsson, Phys. Rev. B 66, 165117 (2002).

[61] A. E. Mattsson, R. Armiento, J. Paier, G. Kresse, J. M. Wills, and T. R. Mattsson, J. Chem. Phys. 128, 084714 (2008).

[62] W. Kohn and A. E. Mattsson, Phys. Rev. Lett. 81, 3487 (1998).

[63] G. L. Oliver and J. P. Perdew, Phys. Rev. A 20, 397 (1979).

[64] J. Tao, J. Chem. Phys. 115, 3519 (2001).

[65] J. Tao, M. Springborg, and J. P. Perdew, J. Chem. Phys. 119, 6457 (2003).

[66] K. Burke, F. G. Cruz, and K.-C. Lam, J. Chem. Phys. 109, 8161 (1998).

[67] T. M. Henderson, B. G. Janesko, and G. E. Scuseria, J. Chem. Phys. 128, 194105 (2008).

[68] J. P. Perdew, S. Kurth, A. Zupan, and P. Blaha, Phys. Rev. Lett. 82, 2544 (1999). 
[69] J. Tao, S. Liu, F. Zheng, and A. M. Rappe, Phys. Rev. B 92, 060401(R) (2015).

[70] A. D. Becke, Int. J. Quantum Chem. 23, 1915 (1983).

[71] L. Miglio, M. P. Tosi, and N. H. March, Surf. Sci. 111, 119 (1981).

[72] I. D. Moore and N. H. March, Ann. Phys. (N.Y.) 97, 136 (1976).

[73] D. C. Langreth and J. P. Perdew, Phys. Rev. B 26, 2810 (1982).

[74] J. Harris and R. O. Jones, J. Phys. F 4, 1170 (1974).

[75] C. Q. Ma and V. Sahni, Phys. Rev. B 20, 2291 (1979).

[76] F. Della Sala and A. Görling, J. Chem. Phys. 115, 5718 (2001).

[77] A. V. Krukau, O. A. Vydrov, A. F. Izmaylov, and G. E. Scuseria, J. Chem. Phys. 125, 224106 (2006).

[78] T. M. Henderson, A. F. Izmaylov, G. E. Scuseria, and A. Savin, J. Chem. Phys. 127, 221103 (2007).
[79] A. V. Krukau, G. E. Scuseria, J. P. Perdew, and A. Savin, J. Chem. Phys. 129, 124103 (2008).

[80] T. M. Henderson, B. G. Janesko, G. E. Scuseria, and A. Savin, Int. J. Quantum Chem. 109, 2023 (2009).

[81] J. H. Skone, M. Govoni, and G. Galli, Phys. Rev. B 89, 195112 (2014).

[82] M. J. Frisch et al., GAUSSIAN development version, revision B.01, Gaussian, Inc., Wallingford, CT, 2009.

[83] J. P. Perdew, M. Ernzerhof, and K. Burke, J. Chem. Phys. 105, 9982 (1996).

[84] Y. Zhao, J. Pu, B. J. Lynch, and D. G. Truhlar, Phys. Chem. Chem. Phys. 6, 673 (2004).

[85] J. Paier, B. G. Janesko, T. M. Henderson, G. E. Scuseria, A. Güneis, and G. Kresse, J. Chem. Phys. 132, 094103 (2010).

[86] B. J. Lynch and D. G. Truhlar, J. Phys. Chem. A 107, 8996 (2003). 